Research Report

\title{
PHENOMENOLOGY OF DELIRIUM IN DECOMPENSATED LIVER DISEASE PATIENTS-A PROSPECTIVE OBSERVATIONAL STUDY
}

\author{
B Lakshmi Dorai ${ }^{1 *}$, G Raghuthaman ${ }^{2}$ \\ ${ }^{1}$ Department of Psychiatry, Vinayaka Mission's Kirupananda Variyar Medical College and Hospital, Salem \\ ${ }^{2}$ Department of Psychiatry, PSG Institute of Medical Sciences \& Research, Coimbatore \\ *Corresponding address: Department of Psychiatry, Vinayaka Mission's Kirupananda Variyar Medical College, Sankari Main \\ Rd, Ariyanur, Salem, Tamil Nadu 636308, India. Email:1akshmidorai.b2@gmail.com
}

\begin{abstract}
Background: Decompensated liver disease (DCLD) is associated with various cognitive changes. The present study aimed to estimate the prevalence, phenomenology, and course of delirium in decompensated liver disease patients. Methods: This prospective observational study was done on 111 patients admitted to the gastroenterology department with the diagnosis of DCLD. Richmond Agitation Sedation Scale (RASS) and confusion assessment method (CAM) were used to detect delirium for the first five consecutive days. Patients detected to be having delirium were administered a delirium rating scale (DRS) for the next seven days to evaluate the features of delirium. Descriptive analysis was carried out by mean and standard deviation for quantitative variables, frequency, and proportion for categorical variables. For normally distributed quantitative parameters, the mean values were compared using an independent sample t-test. Categorical outcomes were compared between study groups using the chi-square test. coGuide version V.1.0 was used for statistical analysis. Results: The prevalence of delirium among patients with DCLD was 34 (30.63\%). All delirious patients continued to have delirium symptoms for the initial three days. Six patients remitted as early as the fourth day of DRS assessment, while nine patients continued to have delirium symptoms even by the seventh day of evaluation. Conclusion: Nearly one-third of patients with DCLD presented with delirium. They presented with insomnia, cognitive deficits, motor retardation, and minimal psychotic symptoms. Many patients completely recovered from delirium within a week, but a few had delirium up to the seventh day.
\end{abstract}

Keywords: delirium, Hepatitis, hepatic encephalopathy, cirrhosis

\section{Introduction}

Delirium is a complex neuropsychiatric disorder and a potentially life-threatening acute brain dysfunction. Among the various causes of delirium, decompensated liver disease (DCLD) is an important one. ${ }^{1}$ The literature on DCLD has dealt in-depth with various cognitive changes associated with it. Various terms are given for it as hepatic encephalopathy, minimal hepatic encephalopathy, episodic and persistent

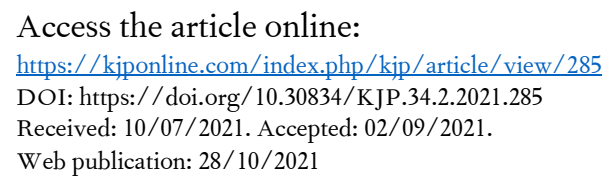

encephalopathy. ${ }^{2}$ During the natural course of DCLD like fatty liver (steatosis), followed by inflammation ofthe liver tissue (hepatitis), scar tissue formation (fibrosis), and finally, destruction of liver architecture (cirrhosis) cause a decrease in the number of functional liver cells resulting in an inability to remove toxic substances from the blood. As a result, ammonia and manganese accumulate in the brain leading to structural

Please cite this article as:. Dorai B L, Raghuthaman G.. Phenomenology of delirium in decompensated liver disease patients-a prospective observational study. Kerala Journal of Psychiatry 2021; 34(2): 
changes in the astrocytes. These factors contribute to altered neurotransmission and brain dysfunction.

Based on psychomotor features, two clinical subtypes of delirium have been described; hyperactive and hypoactive. ${ }^{3}$ Some patients fluctuate between these two and fall into the third subtype called mixed delirium. ${ }^{4}$ Hyperactive delirium is characterized by agitated behaviour with increased psychomotor activity. ${ }^{5}$ Alcohol withdrawal is a classic example of hyperactive delirium. Patients with hypoactive delirium are quiet and lethargic with decreased psychomotor activity. ${ }^{5}$ Probably, these phenotypes signify different forms of neurologic dysfunction. However, as of now, clinically, there is no substantial understanding to pinpoint the aetiology. ${ }^{6}$

The prevalence of cognitive changes among patients with decompensated liver disease (DCLD) ranges from $30 \%-60 \%$. The annual risk of a patient with DCLD developing hepatic encephalopathy is $20 \%$, and $60 \%$ $80 \%$ of the patients with DCLD have some form of cognitive impairment during their lifetime. ${ }^{7}$ Survival probability among DCLD patients who developed encephalopathy was $42 \%$ at one year and $23 \%$ at three years. ${ }^{8}$ Prompt identification and management of this condition reduces mortality, morbidity and also shortens hospital stay.' Literature available includes studies on clinical profile, plasma ammonia levels in patients with liver failure with or without hepatic encephalopathy (HE) and prognostic factors associated with HE. ${ }^{10-12}$ Longitudinal studies are found to be looking into the phenomena of all-cause delirium. ${ }^{13}$ Though there are Indian studies on all-cause delirium ${ }^{14}$, studies looking specifically at hepatic encephalopathy (HE) are rare. Hence, the current study aimed to study the phenomenology and course of delirium in decompensated liver disease patients. The objectives were to estimate the prevalence of delirium in patients admitted with decompensated liver disease and determine the association between delirium with clinical and laboratory parameters.

\section{MATERIALS AND METHODS}

Study population and study site: The study was conducted on patients admitted in the ward and ICU of a PSG Institute of Medical Science and Research, Coimbatore, with decompensated liver disease (DCLD) (based on clinical examination and supportive laboratory test reports).

Study design: Prospective observational study.

Study duration: Eight months from May 2013 to January 2014.

\section{Inclusion Criteria:}

1. Patients who were diagnosed with decompensated liver disease (DCLD).

2. Age between 15 to 80 years.

\section{Exclusion criteria:}

1. Patients who were having severe mental disorders and mental retardation.

2. Patients on ventilators.

3. Patients were having severe impairment in vision (blindness) and hearing as they could not undergo the psychological tests.

Sample size: The sample size was calculated assuming the prevalence of delirium as $20 \%$ as per the study by FF Poordad et al. ${ }^{15}$ The other parameters considered for sample size calculation were $8 \%$ absolute precision and a $95 \%$ confidence level. The following formula was used for sample size calculation.

$$
n=\frac{Z^{2} P(1-P)}{d^{2}}
$$

Where $n=$ Sample size, $Z=\mathrm{Z}$ statistic for the level of confidence, $P=$ Expected prevalence of proportion (if the expected prevalence is $20 \%$, then $P=0.2$ ), and $d=$ Absolute precision (if the precision is $8 \%$, then $d=0.08$ ). The required number of subjects as per the calculation mentioned above was 97. To account for a nonparticipation rate of about $4 \%$ (four subjects), it was decided to sample about 111 subjects into the study.

Sampling method: All the eligible subjects were recruited into the study by consecutive sampling until the sample size was reached.

Ethical considerations: The Institutional Human Ethics Committee, PSG Institute of Medical Sciences \& Research , Coimbatore approved the study, and the proposal approval number is $13 / 064$. Written informed consent was obtained from the patient, and most were not capable of giving informed consent due to episodes of delirium at entry. Consent from the nearest key family member was taken in these cases. 


\section{Data collection tools and clinical examination}

Patient proforma: Consists of data regarding sociodemographic profile, substance use details, clinical and laboratory parameters. A single examiner (psychiatrist) administered all the scales.

\section{Various scales used for measurement:}

\section{SADQ (severity of alcohol dependence questionnaire)}

The Severity of Alcohol dependence questionnaire was developed by Addiction Research Unit at Maudsley Hospital in 1983. It covers five aspects of alcohol dependence like Physical withdrawal symptoms, Affective withdrawal symptoms, relief in drinking, the frequency of alcohol consumption, and the speed with which onset of withdrawal symptoms occurs. It focuses on the last six months of heavy alcohol drinking. It takes 2-5 minutes to administer this questionnaire. ${ }^{16}$

\section{RASS (Richmond Agitation Sedation Scale)}

In our study, we use this scale to assess the level of consciousness of the patient. The Richmond Agitation Sedation Scale was developed at Virginia Commonwealth University in Richmond by a multidisciplinary team, and it can be performed in less than 20 seconds and requires minimal training.

\section{CAM-ICU (confusion assessment method)}

Confusion Assessment Method- ICU was developed by Sharon Inouye et al. in 1990, subsequently revised in October 2010. It is a bedside assessment tool, even usable by non-psychiatrists. This was designed mainly for ICU patients who are critically ill, with or without a ventilator, and unable to talk. It takes 5-8 minutes to assess the presence or absence of Delirium. ${ }^{17}$

The Delirium Rating Scale-Revised 98 (DRS-R-98) and administration of various scales:

The timings for data collection were scheduled between 5-7 pm because it is well known that delirium worsens during evening hours. The severity of alcohol dependence questionnaire (SADQ) was used on patients with a history of alcohol use. Each of the patients was administered RASS and CAM-ICU scales daily for five days to detect delirium. If a patient was detected to be having delirium on any of those five days, from that day onwards DRS-R-98 scale was administered. The onset of delirium was considered as the primary outcome variable. Data collection was scheduled between 5-7 pm of all the seven days of the study period (every 24 hours) to avoid overlap of symptoms and better capture of signs. DRS-R-98 scale is a validated scale with a high inter-rater reliability score ranging from $0.66-0.99$, specificity ranging from $79 \%-88 \%$, and sensitivity of $83 \%-88 \%$. It is a 16 -item clinician-rated scale, and it has two sections. First, 13 items are for assessing delirium symptoms severity, and the next 3 are diagnostic items. First, 13 items are rated from $0-3$ points, and diagnostic items are rated from 0 $2 .{ }^{18}$

Statistical Methods: The onset of delirium was considered as the primary outcome variable. Symptoms at different follow-up periods were considered as the primary explanatory variable. Demographic variables (like age, gender), clinical variables, and laboratory parameters were considered other study-relevant variables. Descriptive analysis was carried out by mean and standard deviation for quantitative variables, frequency, and proportion for categorical variables. For normally distributed quantitative parameters, the mean values were compared using an independent sample ttest. Categorical outcomes were compared between study groups using the chi-square test. P-value $<0.05$ was considered statistically significant. The coGuide version V.1.0 was used for statistical analysis. ${ }^{19}$

\section{RESULTS}

In our study, the prevalence of delirium among patients with DCLD was $30.63 \%$ (34). 94 (84.7\%) were males in the study sample, and $17(15.3 \%)$ were females. More than half of the study group had been diagnosed with alcohol-induced DCLD 64(57.7\%). The majority of patients with DCLD had portal HTN91(82\%), ascites 43(38.7\%), and oesophagal varices 36 (32.4\%) were almost equally distributed in the entire sample. Amongl11 patients, 71 patients (64\%) had a history of alcohol consumption in their lifetime. The number of patients using nicotine in the form of smoking was 48(43.2\%), and in the form of tobacco, chewing was noted only in 10 (9\%) of patients. Among the patients who had a history of alcohol consumption in their lifetime, around 36 (50\%) consumed alcohol within one month before hospital admission. (Table 1)

Patients continued to have sleep disturbance throughout one week of delirium. Sleep disturbances seen were sleep-wake cycle disruption, insomnia, day-night 
reversal, and frequent daytime naps. From the fourth day onwards, psychotic symptoms (delusions, perceptual abnormalities, and thought disorder) started Table 1: Summary of demographic variables and clinical parameters $(\mathrm{N}=111)$

\begin{tabular}{|c|c|}
\hline Petameters & Summary \\
\hline \multicolumn{2}{|l|}{ Gender } \\
\hline Male & $94(84.7 \%)$ \\
\hline Female & $17(15.3 \%)$ \\
\hline Age & $53.6 \pm 8$ years \\
\hline \multicolumn{2}{|l|}{ DCLD subtypes } \\
\hline DCLDalcohol-induced & $64(57.7 \%)$ \\
\hline DCLD-cryptogenic & $15(13.5 \%)$ \\
\hline DCLD- other types & $32(28.8 \%)$ \\
\hline \multicolumn{2}{|l|}{ Manifestations of DCLD } \\
\hline Portal hypertension & $91(82 \%)$ \\
\hline Ascites & $43(38.7 \%)$ \\
\hline Esophageal varices & $36(32.4 \%)$ \\
\hline Mean total bilirubin & $6.58 \pm 0.25$ \\
\hline Mean SGPT & $49.31 \pm 5.33$ \\
\hline Mean SGOT & $84.38 \pm 9.45$ \\
\hline Mean alkaline phosphatase & $133 \pm 20.15$ \\
\hline Mean proteins total & $6.27 \pm 0.18$ \\
\hline \multicolumn{2}{|l|}{ Substance use } \\
\hline Alcohol & $71(64 \%)$ \\
\hline Nicotine (smoking) & $48(43.2 \%)$ \\
\hline Nicotine (Chewable) & $10(9 \%)$ \\
\hline \multicolumn{2}{|l|}{ Last drink of Alcohol consumption $(n=71)$} \\
\hline 0 to 30 days of admission & $36(50.7 \%)$ \\
\hline between 30 to 90 days of admission & $8(11.2 \%)$ \\
\hline$>90$ days of admission & $27(38.0 \%)$ \\
\hline \multicolumn{2}{|c|}{ SADQ severity of alcohol dependence $(\mathrm{no}=51)$} \\
\hline Mild & $3(5.8 \%)$ \\
\hline Moderate & $28(54.9 \%)$ \\
\hline Severe & $20(39.2 \%)$ \\
\hline \multicolumn{2}{|l|}{ The onset of Delirium $(\mathrm{no}=34)$} \\
\hline Day 1 & $23(67.6 \%)$ \\
\hline Day 2 & $7(20.5 \%)$ \\
\hline Day 3 & $4(11.7 \%)$ \\
\hline \multicolumn{2}{|l|}{ Last drink $(\mathrm{N}=71)$} \\
\hline$<7$ days & $26(36.62 \%)$ \\
\hline$>7$ days & $45(63.38 \%)$ \\
\hline
\end{tabular}

Patients continued to have sleep disturbance throughout one week of delirium. Sleep disturbances seen were sleep-wake cycle disruption, insomnia, day-night reversal, and frequent daytime naps. From the fourth day onwards, psychotic symptoms (delusions, perceptual abnormalities, and thought disorder) started reducing and were absent on the sixth and seventh days. On day 7, patients continued to have the following symptoms; sleep disturbance 9 (100\%), motor retardation $4(44.4 \%)$. The cognitive deficits which continued were disorientation, inattention, visuospatial deficits, short-term memory loss, and long-term memory loss. (Table 2)

We looked for association with hepatic encephalopathy by considering various parameters like age, gender, the severity of DCLD presentation, alcohol use, and LFT values. We compared these parameters between delirious and non-delirious patients. There were no statistically significant differences between delirious and non-delirious in various parameters like age, gender, the severity of DCLD presentation, and LFT parameters like (SGPT, SGOT, alkaline phosphatase, total protein, GGT) (p-value >0.05) except for bilirubin, which showed statistical significance. (Table 3)

\section{DISCUSSION}

In our study, the prevalence of delirium in patients with decompensated liver disease was 30.63\%. Among the various causes of decompensated liver disease, alcoholinduced DCLD was seen in most patients (57\%). While analyzing the association, none of the factors like age, gender, alcohol, or severity of liver dysfunction was associated with delirium except for bilirubin in DCLD patients.

The mean age of the participants was $53.6 \pm 8$ years, and $84.7 \%$ were males, and $15.3 \%$ were females. A study on cirrhotic hepatitis $\mathrm{C}$ patients had a mean age of $57.8 \pm 7$ and $63 \%$, and $37 \%$ were males and females, respectively. 20

Our study was comparable to other studies on cognitive changes in decompensated liver disease (30\%-60\%) in the prevalence of delirium. ${ }^{21}$ Overt hepatic encephalopathy occurs in approximately $30 \%-45 \%$ of cirrhotic patients and $10 \%-50 \%$ of patients with the transjugular intrahepatic portosystemic shunt. ${ }^{22}$ Minimal hepatic encephalopathy, characterized by 
Table 2: Course of each symptom of delirium in 7days period

\begin{tabular}{lccccccc}
\hline SL. No & Day 1 & Day 2 & Day 3 & Day 4 & Day 5 & Day 6 & Day 7 \\
\hline Insomnia & $34(100 \%)$ & $29(96.6 \%)$ & $2596.1 \%)$ & $17(94.4 \%)$ & $9(81.8 \%)$ & $10(90.9 \%)$ & $9(100 \%)$ \\
Perceptual isturbance & $10(29.4 \%)$ & $7(23.3 \%)$ & $4(15.3 \%)$ & $2(11.1 \%)$ & 0 & 0 & 0 \\
Delusions & $10(29.4 \%)$ & $7(23.3 \%)$ & $3(11.5 \%)$ & 0 & 0 & 0 & 0 \\
Affect disturbances & $31(91.1 \%)$ & $23(76.6 \%)$ & $1246.1 \%)$ & $10(55.5 \%)$ & $5(45.4 \%)$ & $3(27.2 \%)$ & $1(11.1 \%)$ \\
Language deficits & $18(52.9 \%)$ & $12(40 \%)$ & $4(15.3 \%)$ & $2(11.1 \%)$ & $1(9 \%)$ & 0 & 0 \\
Thought disorder & $11(32.3 \%)$ & $6(20 \%)$ & $4(15.3 \%)$ & $1(5.5 \%)$ & $1(9 \%)$ & 0 & 0 \\
Motor agitation & $18(52.9 \%)$ & $16(53.3 \%)$ & $1038.4 \%)$ & $8(44.4 \%)$ & $5(45.4 \%)$ & $2(18.1 \%)$ & $2(22.2 \%)$ \\
Motor retardation & $32(94.1 \%)$ & $22(73.3 \%)$ & $1661.5 \%)$ & $10(55.5 \%)$ & $5(45.4 \%)$ & $4(36.3 \%)$ & $4(44.4 \%)$ \\
Disorientation & $34(100 \%)$ & $26(86.6 \%)$ & $1453.8 \%)$ & $8(44.4 \%)$ & $4(36.3 \%)$ & $2(18.1 \%)$ & $2(22.2 \%)$ \\
Inattention & $30(88.2 \%)$ & $21(70 \%)$ & $1142.3 \%)$ & $4(22.2 \%)$ & $4(36.3 \%)$ & $2(18.1 \%)$ & $2(22.2 \%)$ \\
Short-term memory & $25(73.5 \%)$ & $15(50 \%)$ & $9(34.6 \%)$ & $5(27.7 \%)$ & $3(27.2 \%)$ & $2(18.1 \%)$ & $2(22.2 \%)$ \\
Long-term memory & $19(55.8 \%)$ & $13(43.3 \%)$ & $6(23 \%)$ & $4(22.2 \%)$ & $1(9 \%)$ & $1(9 \%)$ & $1(11.1 \%)$ \\
Visuo-spatial deficits & $17(50 \%)$ & $12(40 \%)$ & $6(23 \%)$ & $4(22.2 \%)$ & $1(9 \%)$ & $1(9 \%)$ & $1(11.1 \%)$ \\
Total D-pts & 34 & 30 & 26 & 18 & 11 & 11 & $9(26.4 \%)$ \\
\hline
\end{tabular}

Table 3: Comparison of demographic variables and clinical parameters between delirium and non-delirium $(\mathrm{N}=111)$

\begin{tabular}{llll}
\hline Parameters & Delirium $(\mathrm{N}=34)$ & Non-delirium $(\mathrm{n}=77)$ & P value \\
\hline $\begin{array}{l}\text { Mean age } \\
\text { Gender }\end{array}$ & 55.09 & 52.96 & $0.372 \ddagger$ \\
$\quad$ Male & $30(88.2 \%)$ & & \\
Female & $4(11.8 \%)$ & $64(83.1 \%)$ & $0.476 \dagger$ \\
The severity of DCLD presentation & & $13(16.9 \%)$ & \\
$\quad$ Portal HTN & $26(76.5 \%)$ & & $0.422 \dagger$ \\
Esophageal varices & $13(38.2 \%)$ & $65(84.4 \%)$ & $0.382 \dagger$ \\
Ascites & $11(32.4 \%)$ & $23(29.9 \%)$ & $0.404 \dagger$ \\
LFT parameters & & $32(41.6 \%)$ & \\
Total Bilirubin & $8.65 \pm 0.13$ & & $0.010 \ddagger$ \\
SGPT & $38.53 \pm 3.08$ & $5.67 \pm 0.09$ & $0.908 \ddagger$ \\
SGOT & $77.88 \pm 8.90$ & $51.88 \pm 5.11$ & $0.482 \ddagger$ \\
Alkaline Phosphatase & $144.26 \pm 12.33$ & $87.25 \pm 7.78$ & $0.435 \ddagger$ \\
Total Protein & $6.30 \pm 1.01$ & $128.16 \pm 11.45$ & $0.828 \ddagger$ \\
GGT & $96.68 \pm 3.34$ & $6.26 \pm 0.99$ & $0.954 \ddagger$ \\
Last drink & & $141.61 \pm 2.56$ & \\
<7days & $9(34.6 \%)$ & & $0.797 \dagger$ \\
$>7$ days & $14(31.1 \%)$ & $31(68.9 \%)$ & \\
\hline
\end{tabular}

$\ddagger$ Independent sample t-test, $f$ chi-square test

subtle motor and cognitive deficits, affects approximately $20 \%-60 \%$ of patients with liver disease. ${ }^{8}$
The true incidence and prevalence of hepatic encephalopathy is difficult to establish because of 
Figure 1: Course of Delirium as evaluated by Delirium rating scale (DRS)

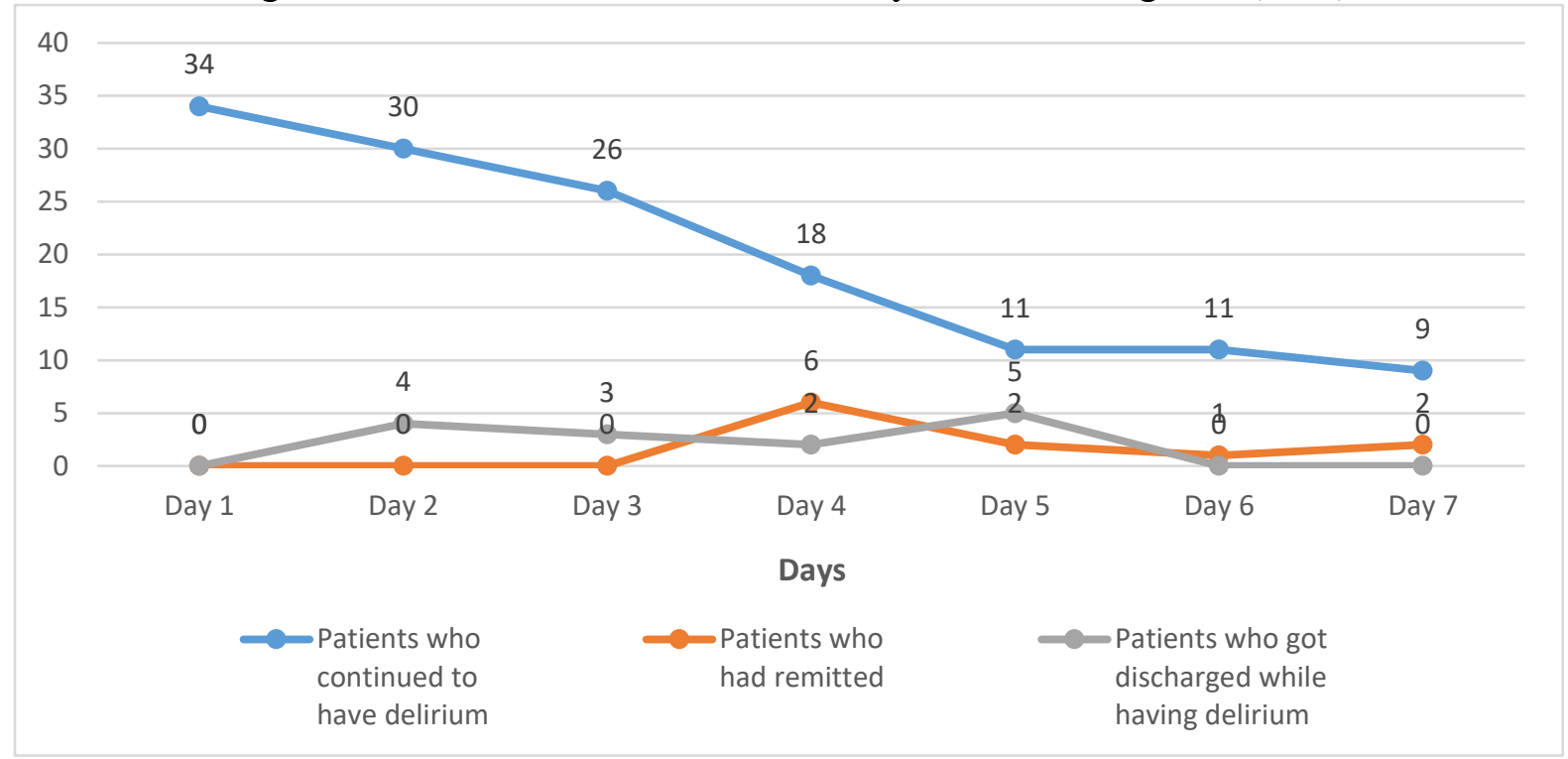

considerable differences in the aetiology and severity of hepatic encephalopathy and the varying tools in diagnosing.

Unlike the previously used scales like West-Haven Criteria, PSE-syndrome test (Portosystemic encephalopathy Syndrome Test and Repeatable Battery for the Assessment of Neuropsychological Status (RBANS) test, which detects cognitive changes rather than delirium, we have used RASS and CAM-ICU, which detect delirium due to any cause. Unlike previous scales (which need physicians and psychologists to assess), nursing staff can administer these scales with minimal time and monetary resources.

To the best of our knowledge, this is the first study to look into the breadth of the phenomenology of delirium in patients with DCLD. Our study describes delirium in detail by tracing out and defining 13 of its symptoms over seven days (Table 3). By unfolding how exactly each of these symptoms remitted in a week, this study also portrays the course of delirium.

On meticulously observing the symptoms of delirium, sleep disturbance is present in all patients, and it is present throughout delirium. Sleep disturbance includes sleep-wake cycle disturbance, day-night reversal, nocturnal disturbance only, or frequent daytime naps. Sleep-wake inversion or the combination of restless nights and excessive daytime sleepiness were first described as a sign of overt HE by Sherlock et al. ${ }^{23}$
Similar findings were recorded in studies with both insomnia and fragmented sleep with multiple night awakening sin cirrhosis patients. ${ }^{24}$

The next glaring symptom is disorientation, where the person is disoriented to time, place, and person in increasing order of severity. While recovering, the patient first gets oriented to person, and the last thing he gets oriented to is time. Almost $100 \%$ of the patient had disorientation on day one. Similar results were shown in studies where almost all participants showed disorientation features. ${ }^{25} \mathrm{~A}$ recent longitudinal study of the phenomenology of delirium showed the prevalence of disorientation in all visits to be $81 \%$ with DRS-R- 98 and $69 \%$ with the cognitive test for delirium (CTD). ${ }^{13}$ Another longitudinal study (Fan et al. 2005) has suggested that psychomotor abnormalities and sleepwake cycle disturbances present mainly in the early course of a delirium. ${ }^{26}$ In contrast, disorientation, inattention, impaired memory, and sleep disturbances are present throughout delirium (McCusker et al., 2003). ${ }^{27}$

Another important area in delirium symptoms is cognitive deficits. Cognitive deficits consist of inattention, memory impairment, visuospatial disturbances. The cognitive symptom was one of the persistent symptoms patients had while getting discharged. Studies were done in the past, giving weightage on how to detect delirium in patients with a 
subclinical presentation, mainly in cognitive deficits. ${ }^{9} \mathrm{~A}$ study done to assess all-cause delirium's phenomenology using the MDAS scale showed an increased prevalence of perceptual disturbances (e.g., hallucinations) and delusions. In contrast, the present study showed less prevalent symptoms for perceptual disturbances and delusions. ${ }^{28}$ The difference may be due to the scales used in both studies and the latter used only for DCLD patients.

Psychotic symptoms experienced were thought disorder, delusions, and perceptual abnormalities. Delusions predominantly seen were persecutory. Perceptual abnormalities ranged from illusions to hallucinations, predominantly auditory. We also found that psychotic symptoms were the first to remit among all the delirium symptoms. More prevalent symptoms such as hallucinations, illusions, and delusions were seen in patients with the activated subtype in a study done by Ross et al. ${ }^{14}$

Delirium was fully remitted in 11 patients within a week, but 14 patients got discharged within seven days while still having some features of delirium. Three of these patients requested discharge for personal reasons, and four patients got discharged against medical advice. The remaining seven patients were discharged from the hospital side when they still had minimal delirious features (like motor retardation and cognitive deficits).

This study also tried to analyze the association of delirium following hepatic encephalopathy with other factors. Age, gender, marital status, socio-economic status, and education did not significantly affect delirium. Moreover, on generally comparing LFT results between delirious and non-delirious patients, patients with delirium showed significantly more deranged bilirubin values. This is also supported by previous studies. $^{29}$

The main strength of our study was that the participants were followed up by a week to assess the course of delirium in decompensated liver disease in detail.

Limitations: We don't have data on patients who had a more extended hospital stay in which delirium could have developed after five days (stopped visiting DCLD patients on the fifth day itself according to our study protocol). We did not consider other parameters associated with delirium, like biochemical parameters (electrolyte, ammonia, arterial blood gas, etc.), cardiac or renal parameters. Also, pharmacotherapy was not assessed, such as benzodiazepines for alcohol withdrawal that could have precipitated encephalopathy in some patients.

Conclusions: We conclude by saying that a significant number of patients having DCLD present with delirium. They present with insomnia, cognitive deficits, motor retardation, and minimal psychotic symptoms. Many patients completely recovered from delirium within a week, but few had delirium for more than one week. No significant association was seen in delirium patients with liver function tests and alcohol consumption except for bilirubin.

\section{Financial support and sponsorship:}

None.

\section{Conflict of interest:}

None declared.

\section{REFERENCES}

1. Mardini H, Saxby BK, Record CO. Computerized Psychometric Testing in Minimal Encephalopathy and Modulation by Nitrogen Challenge and Liver Transplant. Gastroenterology. 2008;135:1582-90.

2. Córdoba J, Sanpedro F, Alonso J, Rovira A. 1H magnetic resonance in the study of hepatic encephalopathy in humans. Metab Brain Dis. 2002;17:415-29.

3. Sanders RD. Delirium, neurotransmission, and network connectivity: The search for a comprehensive pathogenic framework. Anesthesiology. 2013;118:4946.

4. Morandi A, Pandharipande P, Trabucchi M, Rozzini R, Mistraletti G, Trompeo AC, et al. Understanding international differences in terminology for delirium and other types of acute brain dysfunction in critically ill patients. Intensive Care Med. 2008;34(10):1907-15.

5. Meyer $\mathrm{T}$, Eshelman A, Abouljoud $\mathrm{M}$. Neuropsychological Changes in a Large Sample of Liver Transplant Candidates. Transplant Proc. 2006;38(10):3559-60.

6. Reinehr R, Görg B, Becker S, Qvartskhava N, Bidmon $\mathrm{HJ}$, Selbach O, et al. Hypoosmotic swelling and ammonia increase oxidative stress by NADPH oxidase in cultured astrocytes and vital brain slices. Glia. 2007;55(7):758-71.

7. Perry W, Hilsabeck RC, Hassanein T. Cognitive dysfunction in chronic hepatitis C: A review. Dig Dis Sci. 2008;53(2):307-21. 
8. Bajaj JS, Saeian K, Verber MD, Hischke D, Hoffmann RG, Franco J, et al. Inhibitory control test is a simple method to diagnose minimal hepatic encephalopathy and predict development of overt hepatic encephalopathy. Am J Gastroenterol. 2007;102(4):754-60.

9. Bajaj JS. Review article: The modern management of hepatic encephalopathy. Aliment Pharmacol Ther. 2010;31(5):537-47.

10. Butterworth RF. Hepatic encephalopathy - A serious complication of alcoholic liver disease. Alcohol Res Heal. 2003;27(2):143-5.

11. Kundra A, Jain A, Banga A, Bajaj G, Kar P. Evaluation of plasma ammonia levels in patients with acute liver failure and chronic liver disease and its correlation with the severity of hepatic encephalopathy and clinical features of raised intracranial tension. Clin Biochem. 2005;38(8):696-9.

12. Onyekwere CA, Ogbera AO, Hameed L. Chronic liver disease and hepatic encephalopathy: Clinical profile and outcomes. Niger J Clin Pract. 2011;14(2):181-5.

13. Leonard M, Adamis D, Saunders J, Trzepacz P, Meagher D. A longitudinal study of delirium phenomenology indicates widespread neural dysfunction. Palliat Support Care. 2015;13(2):187-96.

14. Ross CA, Peyser CE, Shapiro I, Folstein MF. Delirium: Phenomenologic and Etiologic Subtypes. Int Psychogeriatrics. 1991;3(2):135-47.

15. Poordad FF. The burden of hepatic encephalopathy. Aliment Pharmacol Ther. 2007;25:3-9.

16. Stockwell T, Murphy D, Hodgson R. The Severity of Alcohol Dependence Questionnaire: Its Use, Reliability and Validity. Br J Addict. 1983;78(2):145-55.

17. Inouye SK, Van Dyck CH, Alessi CA, Balkin S, Siegal AP, Horwitz RI. Clarifying confusion: The confusion assessment method: A new method for detection of delirium. Ann Intern Med. 1990;113(12):941-8.

18. Ortiz M, Córdoba J, Doval E, Jacas C, Pujadas F, Esteban R, et al. Development of a clinical hepatic encephalopathy staging scale. Aliment Pharmacol Ther. 2007;26(6):859-67.

19. BDSS Corp. Released 2020. coGuide Statistics software, Version 1.0, India: BDSS corp. Available from: https://www.coguide.in. [Last accessed on 2021 Aug $10]$.

20. Barboza KC, Salinas LM, Sahebjam F, Jesudian AB, Weisberg IL, Sigal SH. Impact of depressive symptoms and hepatic encephalopathy on health-related quality of life in cirrhotic hepatitis C patients. Metab Brain Dis. 2016;31(4):869-80.

21. Bustamante J, Rimola A, Ventura PJ, Navasa M, Cirera I, Reggiardo V, et al. Prognostic significance of hepatic encephalopathy in patients with cirrhosis. J Hepatol. 1999;30(5):890-5.

22. Papatheodoridis G V., Goulis J, Leandro G, Patch D, Burroughs AK. Transjugular intrahepatic portosystemic shunt compared with endoscopic treatment for prevention of variceal rebleeding: A meta-analysis. Hepatology. 1999;30(3):612-22.

23. Sherlock S, Summerskill WHJ, White LP, Phear EA. Portal-Systemic Encephalopathy Neurological Complications of Liver Disease. Lancet. 1954;264:4537.

24. Montagnese S, Middleton B, Skene DJ, Morgan MY. Night-time sleep disturbance does not correlate with neuropsychiatric impairment in patients with cirrhosis. Liver Int. 2009;29(9):1372-82.

25. Grover S, Agarwal M, Sharma A, Mattoo SK, Avasthi A, Chakrabarti S, et al. Symptoms and aetiology of delirium: A comparison of elderly and adult patients. East Asian Arch Psychiatry. 2013;23(2):56-64.

26. Fann JR, Alfano CM, Burington BE, Roth-Roemer S, Katon WJ, Syrjala KL. Clinical presentation of delirium in patients undergoing hematopoietic stem cell transplantation. Cancer. 2005;103:810-20.

27. McCusker J, Cole M, Dendukuri N, Han L BÉ. The course of delirium in older medical inpatients: A prospective study. J Gen Intern Med. 2003;18:696-704.

28. Boettger S, Breitbart W. Phenomenology of the subtypes of delirium: Phenomenological differences between hyperactive and hypoactive delirium. Palliat Support Care. 2011;9(2):129-35.

29. Krieger D, Krieger S, Theilmann L, Jansen O, Gass P, Lichtnecker H. Manganese and chronic hepatic encephalopathy. Lancet. 1995;346:270-4. 